\title{
Wavelet analysis of turbulence in cirrus clouds
}

\author{
S. A. Smith*, P. R. Jonas \\ UMIST, PO Box 88, Manchester, M60 1QD, UK \\ Received: 25 October 1995 / Revised: 4 April 1997 / Accepted: 15 April 1997
}

\begin{abstract}
Two flights of the UK Meteorological Office's Hercules aircraft through daytime frontal cirrus around Scotland have been analysed using wavelet analysis on the vertical velocity time-series from the horizontal runs. It is shown that wavelet analysis is a useful tool for analysing the turbulence data in cirrus clouds. It finds the largest scales involved in producing turbulence, as does Fourier analysis, such as the 2-km spectral peaks corresponding to convective activity during flight A283. Wavelet spectra have the added advantage that the position is shown, and so they identify smaller-scale, highly localised processes such as the production of turbulent kinetic energy by the breaking of KelvinHelmholtz waves due to the vertical shear in the horizontal wind. These may be lost in Fourier spectra obtained for long time-series, though they contribute something to the average spectral density at the appropriate scale. The main disadvantage of this technique is that only octave frequency bands are resolved.
\end{abstract}

\section{Introduction}

Cirrus clouds which form in the upper troposphere are composed mainly of ice and can cover extensive areas. It is necessary to understand better the dynamics of these clouds in order to improve their representation in general circulation models because of the important role they play in the earth's radiation budget. Smallscale turbulence has an important influence on the cloud structure and therefore on the spatial distribution of the optical properties.

In the upper troposphere the thermal stratification is stable, and therefore consumes turbulent kinetic energy (TKE). The sources of turbulence are instabilities

*Currently at: Columbia University and NASA GISS, 2880 Broadway, New York, NY10025, USA associated with gravitational waves and the vertical shear of the horizontal wind, which are neither continuous in time nor spatially homogeneous. Turbulence in the free atmosphere thus occurs intermittently. In the presence of clouds, however, turbulence is also generated by the release of latent heat and radiation effects.

It has been shown by previous observational studies of cirrus clouds, such as those by Dmitriev et al. (1984), Quante and Brown (1992) and Smith and Jonas (1997), which will subsequently be referred to as SJ, that the occurence of turbulence is intermittent or patchy and that the velocity field is anisotropic, leading to a twodimensional character of the flow. In general, turbulence is weak, except for patches where, for example, there is a strong wind shear or convective activity. Even then, the turbulent kinetic energies observed are much smaller than those found in boundary-layer clouds. Furthermore, frontal cirrus tends to be less energetic than jet stream cirrus.

Fourier analysis has been used in the past for the analysis of signals for their frequency content. It can be viewed as a rotation in function space from the time (or space) domain to the frequency domain, which contains sines and cosines as basis functions. These basis functions are localised in frequency only. The disadvantage of Fourier spectra is that the Fourier modes are infinite in physical space and therefore information on the position of local events is lost. This makes the spectra difficult to interprete for data sets which contain different local processes. A local oscillation will contribute to the average Fourier transform, but its location will be lost, as will its amplitude.

Wavelet analysis is a relatively new technique for analysing data into its frequency components which, despite some similarities, holds several advantages over the more traditional Fourier analysis. Both wavelet analysis and Fourier analysis are linear operations which can be viewed as a rotation in function space. In the case of the wavelet transform, however, the frequency domain contains basis functions called wavelets. Wavelets are a set of self-similar functions which 
are localised in frequency and in space, and so their dilations and translations provide simultaneous resolution in scale and position. It is thus possible to isolate local events which produce TKE.

Wavelet bases also have the desirable quality of effectively providing a varying window size: low-frequency wavelets are long and high-frequency wavelets are short, enabling us in theory to isolate very small events and large events easily.

There are infinitely many possible sets of wavelets. The various sets have different trade-offs between how compactly they are localised in space and how smooth they are. The simplest is the Haar basis (see Meneveau, 1991) which is very well localised in physical space. It is desirable to use wavelets which are smoother and have better locality in Fourier space to enable us to identify the dominant frequencies. Such functions have been discovered relatively recently. Daubechies (1988) derived wavelets which satisfy these requirements and are orthonormal to their own translates and dilations. Daubechies wavelets have a fractal structure, and they include both highly localised wavelets and highly smooth wavelets. The simplest and most localised are those with four coefficients.

The aim of the current research is to investigate the use of wavelet analysis to analyse turbulence data obtained within frontal cirrus clouds. Time-series of the vertical velocities measured in cirrus clouds during straight and level horizontal runs from two flights of the Hercules aircraft (UK Meteorological Office) will be analysed. In Sect. 2 we will describe the analysis procedures used, Sect. 3 will describe the details of the observations made, and Sect. 4 will discuss the results. Section 5 contains the conclusions.

\section{Data analysis}

\subsection{Calculation of power spectra}

The power spectra of the vertical velocity $(w)$ variations from horizontal runs of the Hercules were calculated for sections of $256 \mathrm{~s}$ long and contained 8192 data points at the sample frequency of $32 \mathrm{~Hz}$ (a length of almost $40 \mathrm{~km}$ ). The method used is the same as that described in SJ, but a brief description will be given here. The bestfit straight line was removed from the initial time-series, as mean vertical velocities are unreliable. Then $10 \%$ of the data was tapered at both ends using the split cosine bell, to reduce leakage to neighbouring frequency bins. The NAG Fortran Library routine G13CBF was utilised to calculate a smoothed sample spectrum using spectral smoothing by the trapezium frequency (Daniell) window.

Any frequency $f$ in $\mathrm{Hz}$ corresponds to a scale $\lambda$ given by $\lambda=U / f$, where $U$ is the wind speed relative to the aircraft (true air speed, or TAS). The air speed varies with height from $100 \mathrm{~m} \mathrm{~s}^{-1}$ at low levels to $145 \mathrm{~m} \mathrm{~s}^{-1}$ at the highest levels.

As stated in SJ, care has to be taken when analysing these vertical velocity power spectra due to the low- energy densities encountered at such high levels. Quante et al. (1995) observed that all of the $w$ spectra in weak turbulence segments inspected from the Hercules data appeared to be affected by red noise at frequencies higher than about $0.1 \mathrm{~Hz}$, the origin of which was unknown. No corrections were made to the data to eliminate the noise, but it was kept in mind during the spectral analysis. It was shown in SJ that the spectra observed in these cases were not affected by this red noise.

\subsection{Wavelet transform}

The reader is referred to the literature for a detailed description of the wavelet transform, for example Daubechies (1988), Meneveau (1991), Press et al. (1992) and Newland (1993), but a brief description will be given here. The goal of the wavelet transform is to decompose a signal $f(x)$ into its frequency components at different positions. It is convenient to limit the range of $x$ to the unit interval, so that $f(x)$ is assumed to be defined only in the interval $0 \leq x \leq 1$. Thus $x$ is a nondimensional variable. If the signal has a duration in time $t$ of $T$, then $x=t / T$. This signal is assumed to be one cycle of a periodic function, and wavelets are wrapped around this interval if they extend beyond its limits.

We use the discrete wavelet transform (DWT) to decompose a signal which has been sampled at equally spaced intervals. In this case, the signal is the vertical velocity which has a sampling frequency of $32 \mathrm{~Hz}$. The number of datapoints is given as $N=2^{n}$, where $n$ is an integer. The time-series are first detrended as for the Fourier analysis in SJ.

To avoid problems with the non-periodic boundaries of data segments, a flat-topped window was applied to the time-series, as described by Meneveau (1991). To analyse a signal of length $N$, an additional $N / 2$ datapoints from the original data set are added onto each end, forming a signal of length $M=2 N$. (So instead of reading in a time-series of length $N$, a series of length $2 N$ is read into the program). The discrete window used is $z[i]$ :

$$
\begin{aligned}
z[i]=\frac{1}{2}\left[1+\cos \left(\frac{2 \pi i}{N}\right)\right], & i=-\frac{N}{2}+1, \ldots, 0 \\
\text { and } \quad & i=N+1, \ldots, \frac{3}{2} N \\
z[i]=1, \quad i & =1, \cdots, N .
\end{aligned}
$$

The signal is multiplied by $z[i]$, and the discrete wavelet transform is performed on all of these $M$ points, but only the points corresponding to the original time-series are included in the subsequent analysis (this is the flat part of the window). The windowing technique therefore does not affect the part of the time-series of interest.

The family of functions, $\Psi_{j, k}$, used to decompose the signal, is generated from the "mother wavelet" $\Psi$ by dilations and translations described by

$\Psi_{j, k}(x)=2^{j / 2} \Psi\left(2^{j} x-k\right)$, 
where $j$ and $k$ are integers. The integer $j$ is the variable scale index and $k$ is the variable position index. The dilations are discrete and are spaced in octaves, and the translations depend on the scale. Therefore at smaller scales the spatial resolution increases but the resolution in Fourier space decreases, due to the uncertainty principle. However, the separation in frequency compared to the actual frequency remains the same. These are orthogonal Daubechies wavelets (Daubechies, 1988).

Figure 1 demonstrates what Daubechies wavelets with 20 coefficients look like. Two different scales are shown, with the smaller-scale wavelet displaced relative to the other. (The mother wavelet is just the same, but is wider and has a smaller amplitude). This demonstrates not only the shape of the wavelet used, but also how the window size varies with the wavelet scale. These wavelets with 20 coefficients are smoother and therefore better localised in frequency space than those with fewer coefficients. The wavelet coefficients $A_{j, k}$ of the signal $f(x)$ are given by the convolution

$A_{j, k}=\int_{-\infty}^{\infty} f(x) \Psi_{j, k}^{*}(x) \mathrm{d} x$,

where * denotes the complex conjugate.

The DWT involves applying the wavelet coefficient matrix given in Press et al. (1992) hierarchically to a data array containing equally spaced samples of a function, first to the full data vector of length $M$ in this case, then to the "smoothed" vector of length $M / 2$ (which has now had some detail taken out) and so on, progressively taking out more and more detail. This is called a pyramidal algorithm. The output from this is an array of the same length $(M)$ as the input:

$A=\left[a_{0}, a_{1}, a_{2}, a_{3}, a_{4}, a_{5}, \ldots\right]$,

which contains the amplitude of each contributing wavelet to the signal. The first coefficient $a_{0}$ corresponds to level $j=-1$ which is constant, $a_{1}$ corresponds to the single wavelet in level $j=0, a_{2}$ and $a_{3}$ correspond to the two wavelets at level $j=1$ with the second wavelet displaced $1 / 2^{j}$ in the unit interval relative to the first, and so on to the highest level $j=n-1$ which corresponds to the smallest scale.

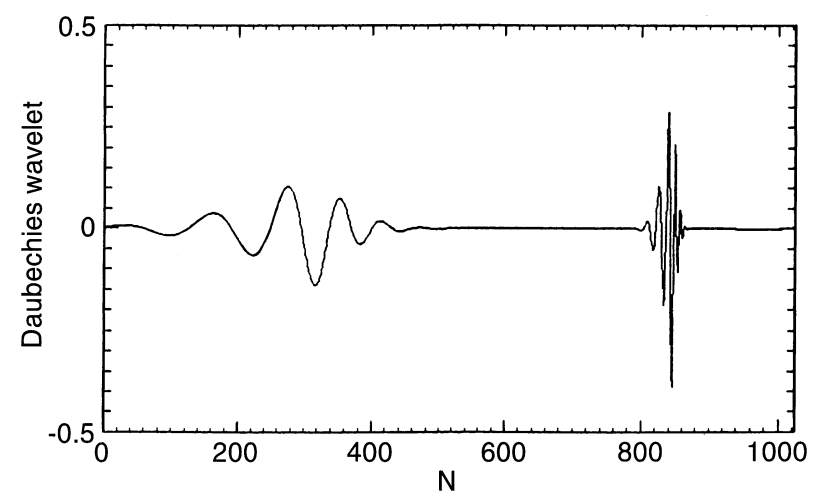

Fig. 1. Daubechies wavelets with 20 coefficients at two different scales and positions. The $x$-axis shows position and the $y$-axis shows the wavelet amplitude
For a signal of length $N=2^{n}$, there are $n+1$ wavelet levels including levels $j=-1$ and 0 . As a signal twice this length was actually transformed, the first two levels correspond to the average and to one wavelet over a time-series twice as long as the required time-series. We know that scales larger than $5 \mathrm{~km}$ (corresponding to $j<5$ ) are unimportant in these data sets from the Fourier analysis carried out in SJ. Therefore only levels $j>3$ will be shown. The horizontal scales and frequency bands corresponding to each level are shown in Table 1, along with the number of wavelets at each level. At each level $j$, there are $k=0: 2^{j}-1$ wavelets, each displaced $M / 2^{j}$ apart in space.

The results are displayed as mean-square maps. The values of the wavelet amplitudes are squared and plotted on a grid framework on which the axes are level $j$ and position such that the volume under the resulting surface represents the mean-square value

$$
\begin{aligned}
\int_{0}^{1} f^{2}(x) \mathrm{d} x= & a_{0}^{2}+a_{1}^{2}+\frac{1}{2}\left(a_{2}^{2}+a_{3}^{2}\right) \\
& +\frac{1}{4}\left(a_{4}^{2}+a_{5}^{2}+a_{6}^{2}+a_{7}^{2}\right)+\ldots
\end{aligned}
$$

and therefore this maps the distribution of the mean square of the function $f(x)$ between wavelets of different level and different position, as described by Newland (1993). This represents the distribution of the total energy over all the scales and positions. Each squared wavelet amplitude is located at a position which corresponds to the centre of the corresponding wavelet.

Wavelet energy spectra are calculated from the DWT by averaging the squared wavelet amplitudes over all positions at each scale, and normalizing by the number of data points in the original time-series. The standard deviations of the individual values about the mean energy is also calculated.

\section{Observations}

Observations were made in relatively thick cirrus during the daytime using the instrumented Hercules aircraft of

Table 1. Correlation between frequency bands and scales for each

\begin{tabular}{|c|c|c|c|}
\hline Level J & no. waves & $\begin{array}{l}\text { frequency band } \\
(\mathrm{Hz})\end{array}$ & scale \\
\hline-1 & constant & constant & constant \\
\hline 0 & 0.5 & $0.00098-0.00195$ & $146-73 \mathrm{~km}$ \\
\hline 1 & 1 & $0.00195-0.0039$ & $73-36.6 \mathrm{~km}$ \\
\hline 2 & 2 & $0.0039-0.0078$ & $36.6-18.3 \mathrm{~km}$ \\
\hline 3 & 4 & $0.0078-0.0156$ & $18.3-9.2 \mathrm{~km}$ \\
\hline 4 & 8 & $0.0156-0.0313$ & $9.2-4.6 \mathrm{~km}$ \\
\hline 5 & 16 & $0.0313-0.0625$ & $4.6-2.3 \mathrm{~km}$ \\
\hline 6 & 32 & $0.0625-0.125$ & $2.3-1.16 \mathrm{~km}$ \\
\hline 7 & 64 & $0.125-0.25$ & $1.16-572 \mathrm{~m}$ \\
\hline 8 & 128 & $0.25-0.5$ & $572-286 \mathrm{~m}$ \\
\hline 9 & 256 & $0.5-1.0$ & $286-143 \mathrm{~m}$ \\
\hline 10 & 512 & $1.0-2.0$ & $143-71 \mathrm{~m}$ \\
\hline 11 & 1024 & $2.0-4.0$ & $71-36 \mathrm{~m}$ \\
\hline 12 & 2048 & $4.0-8.0$ & $36-18 \mathrm{~m}$ \\
\hline 13 & 4096 & $8.0-16.0$ & $18-9 \mathrm{~m}$ \\
\hline
\end{tabular}
wavelet level J. Also shown is the number of wavelets at each scale 
the Meteorological Research Flight, as part of the EUCREX (European Cloud and Radiation Experiment) project. The standard instrumental performance has been described by Nicholls (1978) and Slingo et al. (1982), among others.

The air velocities relative to the aircraft were found from the pitot-static system and the freely rotating wind vanes, at a frequency of $32 \mathrm{~Hz}$. The inertial navigation system (INS) was used to transform these into the stationary-earth-based coordinate system.

\subsection{Flight $A 283$}

Flight A283 took place between 09:05 and 16:40 on 24 September 1993. A waving cold front, associated with a low-pressure system north of Iceland, was moving slowly from west to east across the British Isles. Surface winds were roughly south-westerly during the period.

The flight was carried out in the leading edge of a thick sloping sheet of cirrus in the region of Wick in Scotland. There was thick cirrus in the west of the operating region and the leading cirrus edge was to the east of the region. For the most part, there was no significant mid-level cloud (although there was some mid-level cloud in the west of the region). The main cirrus layer was between 6.4 and $10.4 \mathrm{~km}$, although there were some patches of cirrus extending even above $11 \mathrm{~km}$ in places. The heights of cloud base and cloud top varied along a line from west to east, but the cirrus deck was between 3 and $4 \mathrm{~km}$ deep. The very top of the cirrus was unattainable by the aircraft.

Two fixed ground positions A and B were selected, orientated west to east, with B just east of the leading edge of the cirrus. The aircraft performed straight and level runs at several heights between $\mathrm{A}$ and $\mathrm{B}$, travelling at its optimum air speed of between 120 and $145 \mathrm{~m} \mathrm{~s}^{-1}$ (depending on height). The lengths of these horizontal runs were typically $100 \mathrm{~km}$. Profiles were carried out before (ascent) and after (descent) the horizontal runs.

Only the first nine horizontal runs and the first six profiles are used in the subsequent data analysis (before 14:00 GMT), as the INS data was erroneous in the second half of the flight, resulting in unreliable vertical velocity data.

Figure 2 a shows profiles of $2 \mathrm{D}-\mathrm{C}$ ice water content $\left(Q_{I}\right)$, equivalent potential temperature $\theta_{e}$ and the horizontal wind components $u$ (eastward) and $v$ (northward) at the start of the flight. The level at which each level run was flown is indicated on the right-axis by lines labelled by the run numbers. The ice water content in the diagram goes down to zero at the top of the profile sooner than expected because the aircraft flew out of the east edge of the cirrus before reaching cloud top.

\subsection{Flight $A 290$}

Flight A290 took place between 09:30 and 16:40 on 9 October 1993. A low-pressure system was situated over south-west England. An occluded frontal system associated with this was present to the north-west of
Scotland, giving rise to a large amount of cirrus and mixed phase clouds.

The flight was carried out in a thick cirrus layer lying to the north of Scotland. Initially, cirrus cloud-base height was $6.2 \mathrm{~km}$ and cloud-top height was $9.2 \mathrm{~km}$, with some lower-level cloud. However, the cloud deepened and by the end of the flight the base was at a height of $3.5 \mathrm{~km}$. It seemed to have merged with the lower-level cloud. Therefore, on average, the depth of the main cirrus layer was approximately $4 \mathrm{~km}$. The main cirrus layer appeared horizontally uniform through most of the flight.

A profile ascent was flown at the start of the flight from $50 \mathrm{~m}$ up to $9000 \mathrm{~m}$, but there was still thin cirrus above this level. Straight and level runs were then carried out at a number of levels within and below the cloud. These runs were not always flown directly above each other, but the general direction of the runs was west to east. A final profile descent was carried out from $10000 \mathrm{~m}$ (above the cirrus) to $50 \mathrm{~m}$.

Figure $2 \mathrm{~b}$ shows the variation of $Q_{I}, \theta_{e}, u$ and $v$ with height for the first profile of flight A290. There are discontinuities in some of the profiles because a shorter, higher profile is shown above the main profile. The level at which each level run was flown is indicated on the right-axis by lines labelled by the run numbers. The cirrus is above $6300 \mathrm{~m}$, with thicker cirrus above $7100 \mathrm{~m}$.

\section{Results of wavelet analysis}

\subsection{Flight $A 283$}

Figure 3a shows a section of the vertical velocity timeseries taken from run 1 (at a height of $8.9 \mathrm{~km}$ ), of length $N=8192=2^{13}(36 \mathrm{~km})$. Only the section corresponding to the flat part of the data window is shown. Figure $3 \mathrm{~b}$ shows the logarithm (to base 10) of the squared wavelet amplitudes for that time-series, and Fig. $3 \mathrm{c}$ shows the squared wavelet amplitudes normalised at every scale so that the maximum at each scale is 1 . These are shown as grey-scale images, with the largest wavelet amplitudes shown in white. Distance along the flight leg is shown along the $x$-axis. The left-hand $y$-axis is labelled by level number $j$ from 3 to 13. Each of these levels corresponds to an octave band of frequencies shown on the right-hand $y$-axis, from $0.0078 \mathrm{~Hz}$ to the Nyquist frequency $16 \mathrm{~Hz}$ (corresponding to scales between $18.6 \mathrm{~km}$ and $9 \mathrm{~m}$, using a TAS of $144 \mathrm{~m} \mathrm{~s}^{-1}$ ). Table 1 gives the corresponding scales.

From Fig. 3b, local peaks occur at around $j=6$, corresponding to scales between 2.3 and $1.16 \mathrm{~km}$. There are also secondary peaks at smaller scales to around $j=8$, corresponding to scales between 560 and $280 \mathrm{~m}$. Much is also happening at smaller scales, with many small-scale local peaks.

From Fig. 3c we can see how the maxima at each scale vary in position. It can be seen that the maxima at the largest scales do not necessarily correspond in position with these at the smaller scales. This indicates 

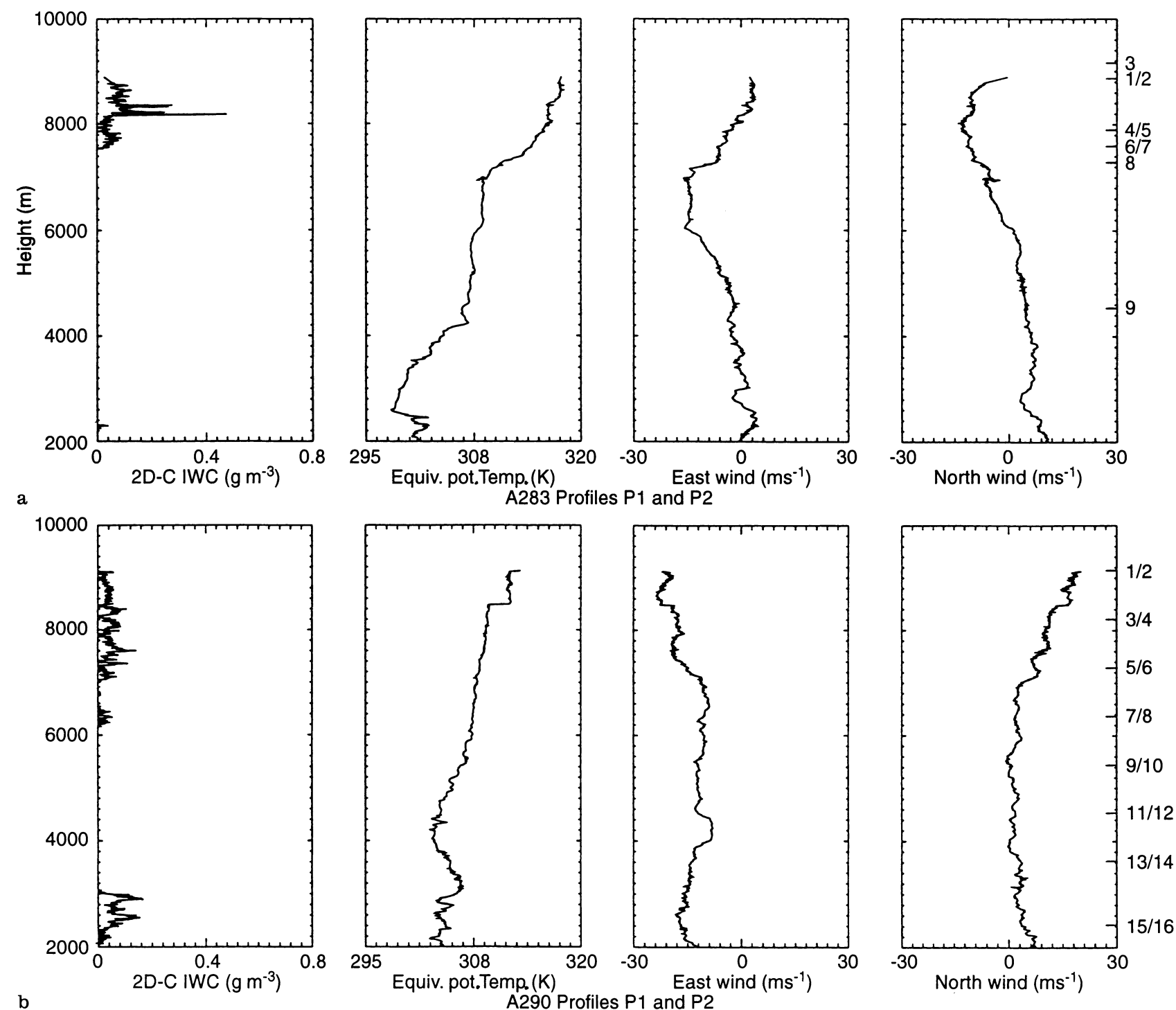

Fig. 2. a Conditions encountered during flight A283. The variation of 2D-C IWC $\left(\mathrm{g} \mathrm{m}^{-3}\right)$, equivalent potential temperature $(\mathrm{K})$, the eastward wind component $u$ and the northward wind component $v$ $\left(\mathrm{m} \mathrm{s}^{-1}\right)$ with height at the beginning of the flight are shown. The height at which each run was flown is marked on the right-hand axis

of the last profile. b Conditions encountered during flight A290. The discontinuity at $8.5 \mathrm{~km}$ in the profiles is due to combining the main profile with a shorter one at higher levels. The height at which each run was flown is marked on the right-hand axis of the last profile

that there are indeed processes other than those at the largest scales which produce TKE. Energy is seen to be handed down from the scales at which shear produces TKE to the smallest scales by the line of maxima in the lower left-hand side of the wavelet transform.

A second, slightly less turbulent region was also analysed for this height, and the maximum of the wavelet amplitudes was less in this case, as should be expected. As the results are otherwise similar to the first section, they are not shown.

Figure 4 shows a section of the vertical velocity timeseries from a run lower in the cloud (run 6), at $7.6 \mathrm{~km}$, with the corresponding mean-square map displaying the logarithm of the squared wavelet amplitudes. Largescale peaks at $j=3$ correspond to wave-like features in the vertical velocity after the first part of the time-series. The more turbulent regions are associated with peaks at

levels $j=5$ and 6 , and have higher wavelet amplitudes at small scales.

\subsection{Flight A290}

A second flight was also analysed, flight A290. The cirrus observed on this day was more homogeneous in the horizontal as seen in the $2 \mathrm{D}-\mathrm{C}$ ice water contents (see SJ). In our previous paper it was noted that the spectral densities for the vertical velocities were much lower than the horizontal velocity spectral densities, indicating that the turbulence was mostly two-dimensional in this case. They were also lower than the vertical velocity spectral densities from flight A283.

Figure 5 a shows a section of the vertical velocity time-series taken from run 3 (at a height of $8.2 \mathrm{~km}$ ) in 

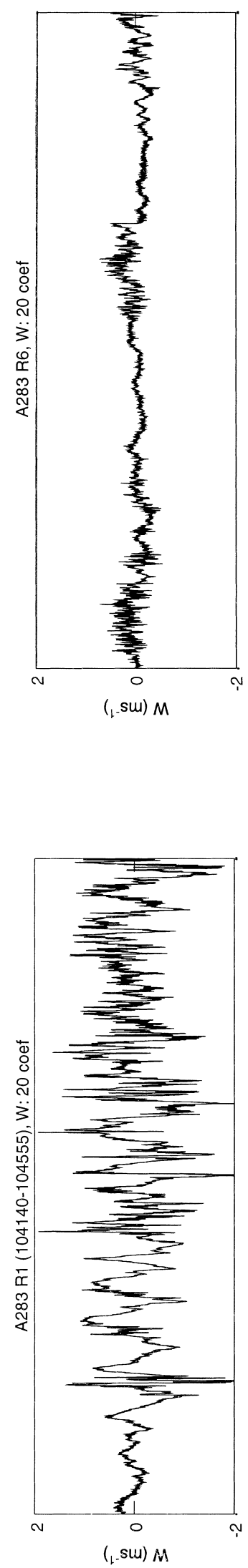

손.

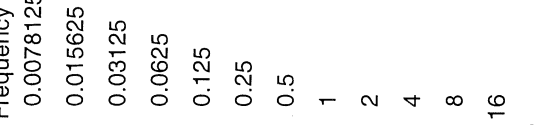

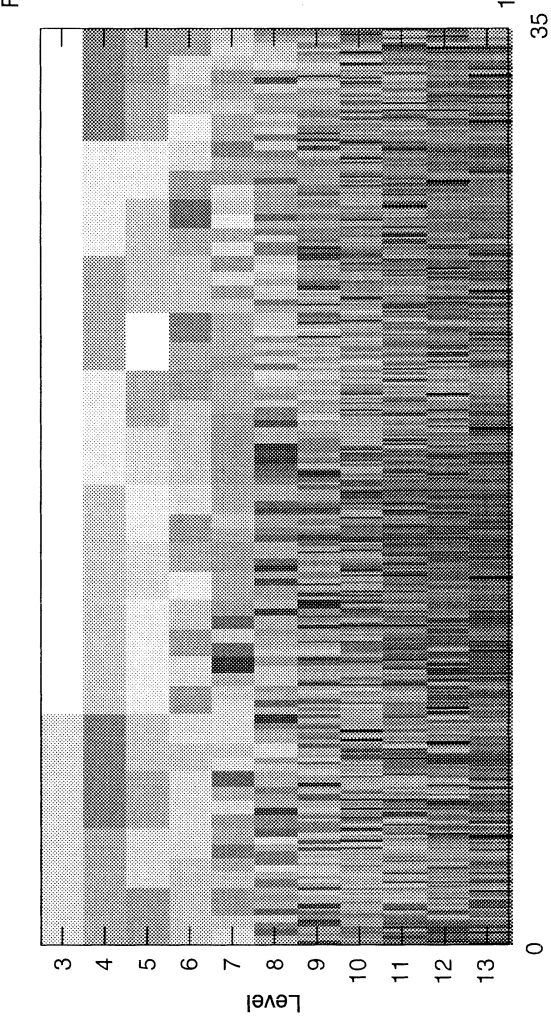

존
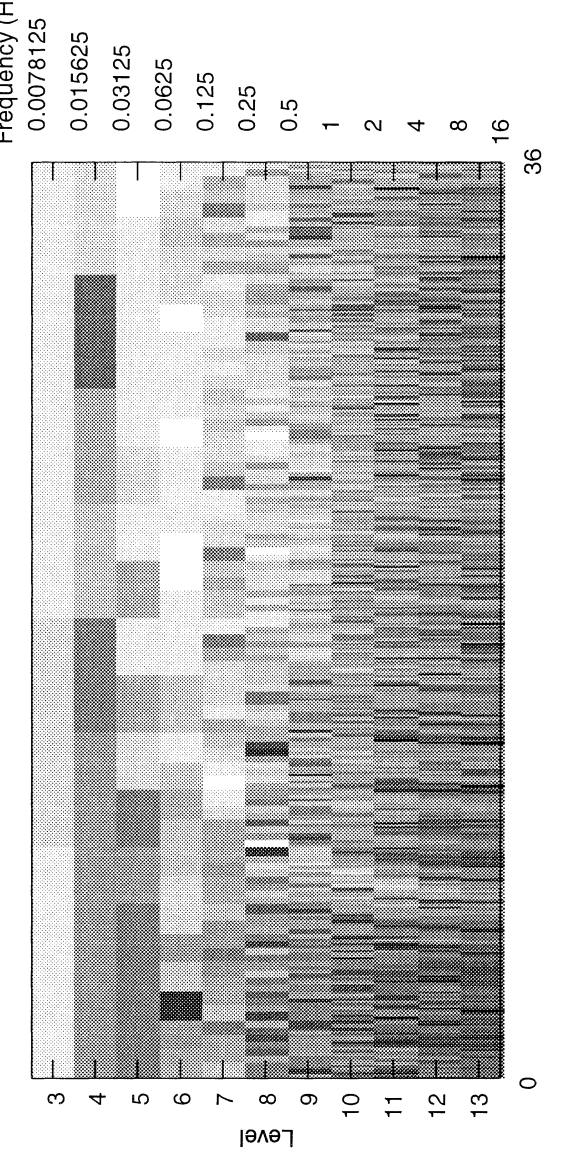

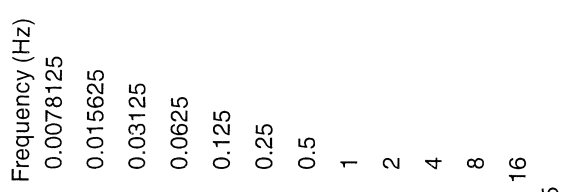

总兽营

政

8 的莎

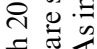

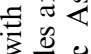

送

㲾表

党雪

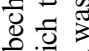

墕

की की

西

bo

要要要

范要

는 넝

욤.

흘 気

堐

웡

政

s.

응흐

过

다에

के $\frac{0}{2}$

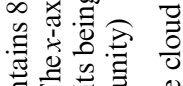

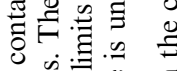

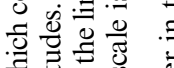

उ. $=0$, है

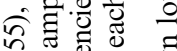

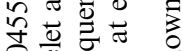
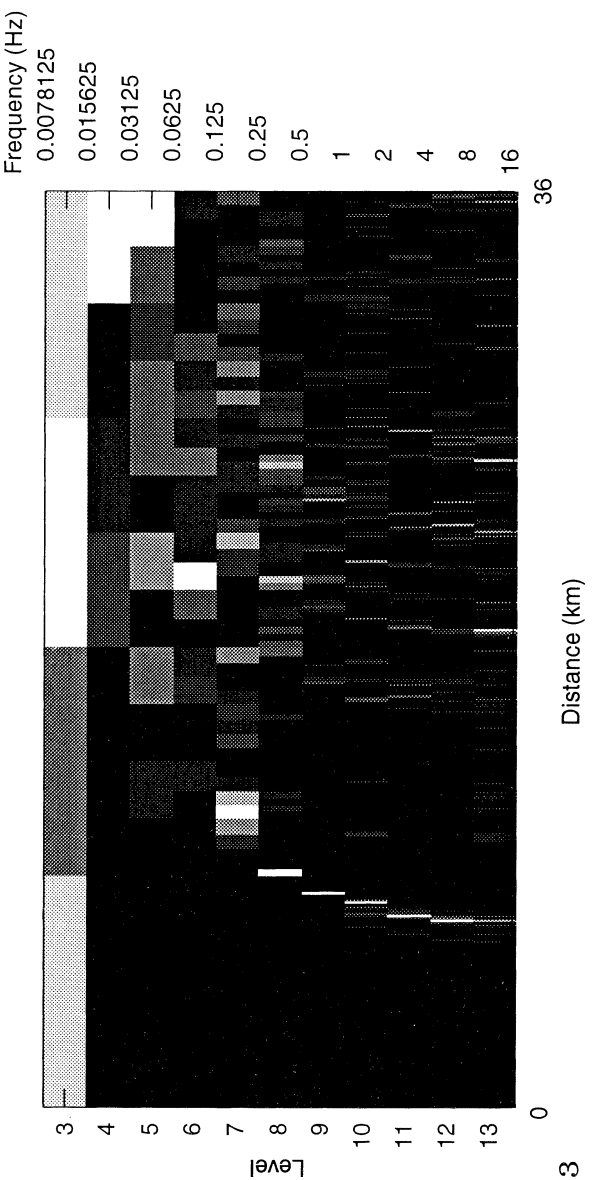

음욜

称氞言

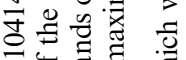

诺

过兽总

해융

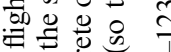

엉

Qิ응

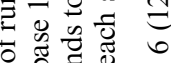

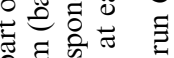

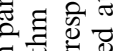

हี:

论

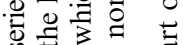

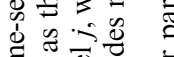

可造记

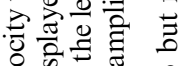

응

可

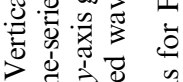

$>$ 竞焉

的品 票

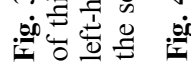


the upper part of the main cloud deck, again of length $N=8192=2^{13}$. Part $\mathrm{b}$ is the mean-square map showing the logarithm (to base 10) of the squared wavelet amplitudes and part $\mathrm{c}$ shows them normalised. This flight was shown by SJ to be much less turbulent than flight A283, and this can be seen in the vertical velocity time-series as compared to the previous cloud-top run, and it is reflected in the fact that the maximum wavelet amplitude is much smaller (this is not obvious from the plot shown because the maximum value in each diagram is always shown as white). The first part is the most turbulent, and here the local peaks occur at varying scales, mostly smaller than $2.3 \mathrm{~km}$ and extending down to $j=9$ or $140 \mathrm{~m}$. The first part is obviously the most turbulent at all scales smaller than $2.3 \mathrm{~km}$ from Fig. 5c.

Figure 6 shows the results for a section of run 1 $(9.2 \mathrm{~km})$ in thin cloud above the main cirrus cloud top. Apart from maxima at the largest scales due to wavelike motion, not much is happening.

\subsection{Comparison between wavelet and Fourier spectra}

The entire detrended vertical velocity time-series from run 1 of flight A283 was shown in SJ with the Fourier spectra for various $100-\mathrm{s}(14-\mathrm{km})$ regions. The spectral densities obtained from each section varied by at least an order of magnitude. In the spectra from the most turbulent regions, there were pronounced peaks at a scale of $2 \mathrm{~km}$. In one of these sections (D) there appear to be small-scale peaks between 600 and $180 \mathrm{~m}$. These were shown to be real peaks.

Figure $7 \mathrm{a}$ shows the Fourier spectrum calculated from the data shown in Fig. 3, which includes the most turbulent sections (C and D) of run 1 from SJ. The peak energy is still observed in the Fourier spectrum at a scale of about $2 \mathrm{~km}$. The four smaller-scale peaks between 600 and $180 \mathrm{~m}$ have been smoothed out in comparison to those observed for run 1 section D in SJ, due to the longer time-series used (corresponding to a length of $36 \mathrm{~km}$ instead of $14 \mathrm{~km}$ ). However, there is still a slight peak at around the 400-m scale. So without dividing the time-series into much shorter sections, peaks due to more localised activities in the vertical velocities can be lost. The spectral slope is approximately $-5 / 3$ as expected for an inertial subrange in regions of isotropic three-dimensional turbulence.

For comparison, the wavelet spectrum is shown in Fig. 7b. The crosses show the average spectrum plus the standard deviation of the energy values across the timeseries. This average spectrum also shows a general slope of $-5 / 3$ and a spectral peak somewhere between 2.3 and $1.16 \mathrm{~km}$. There is also a peak around $400 \mathrm{~m}$. The results from the wavelet analysis therefore agree quite well with the results of the Fourier analysis, especially considering that the resolution in frequency is not as good. The standard deviations are at least of the same order of magnitude as the average energy itself, showing the inhomogeneous nature of the energy field in this cirrus cloud.
It was suggested in SJ that the peaks in the Fourier spectra at scales of around $2 \mathrm{~km}$ were due to convective activity created by cloud-top radiative cooling. The resulting cellular structures were observed in the ice water contents. It was also suggested that the small-scale peaks observed at scales of a few hundred metres were due to the TKE produced by shear instabilities. In a layer of the atmosphere with a stable stratification and a vertical shear in the horizontal wind, Kelvin-Helmholtz waves form. If the wind shear becomes great enough to overcome the stability of the layer (i.e. the Richardson number falls below its critical value of 0.25 ), then these waves become unstable and break to produce turbulence. The wavelength which becomes unstable first as the Richardson number falls below 0.25 depends on the depth of the layer, and in this case is around a few hundred metres.

Figure 8a shows the Fourier spectrum for the data shown in Fig. 6 for run 1 of flight A290. As in SJ, the Fourier spectrum shows a peak at a scale of about $100 \mathrm{~m}$. This has not been averaged out because it appears to be present through most of the time-series.

Figure $8 \mathrm{~b}$ shows the average wavelet spectrum for the same time-series. This also shows a peak at a scale of $100 \mathrm{~m}$, although it is not quite as well defined due to the poor frequency resolution. The standard deviations are again of the same order of magnitude of the average energy. Both the average energy and the standard deviation of the energy along the time-series are smaller than for flight A283, as this cirrus was less turbulent.

In SJ the vertical velocity power spectra flattened out at the larger scales, with no obvious peaks. No evidence for significant cellular convection has been found, with fairly homogeneous ice water content time-series and small standard deviations in the temperature data. This behaviour is observed for most of the cirrus cloud. Smaller-scale peaks were sometimes observed at scales of around $100 \mathrm{~m}$ corresponding to shear TKE production.

\section{Conclusions}

Wavelet analysis has proved to be a useful tool for analysing aircraft data, particularly in the case of cirrus clouds in which the occurence of turbulence is patchy and weak due to the local nature of the TKE production mechanisms. It has been used to identify the scales at which TKE is produced in the cirrus clouds sampled, and the results agree quite well on the whole with the findings in SJ using Fourier techniques.

For flight A283 TKE is produced at scales of about $2 \mathrm{~km}$ by the convective activity set up by cloud-top radiative cooling as well as latent heat effects. Energy is also produced locally by shear generation or, more precisely, by the breaking of Kelvin-Helmholtz waves in layers for which the shear is great enough to overcome the stable lapse rate. This occurs at smaller scales of around a few hundred metres. For flight A290, the production of energy by the Kelvin-Helmholtz instability also occurs but in the absence of convection. 

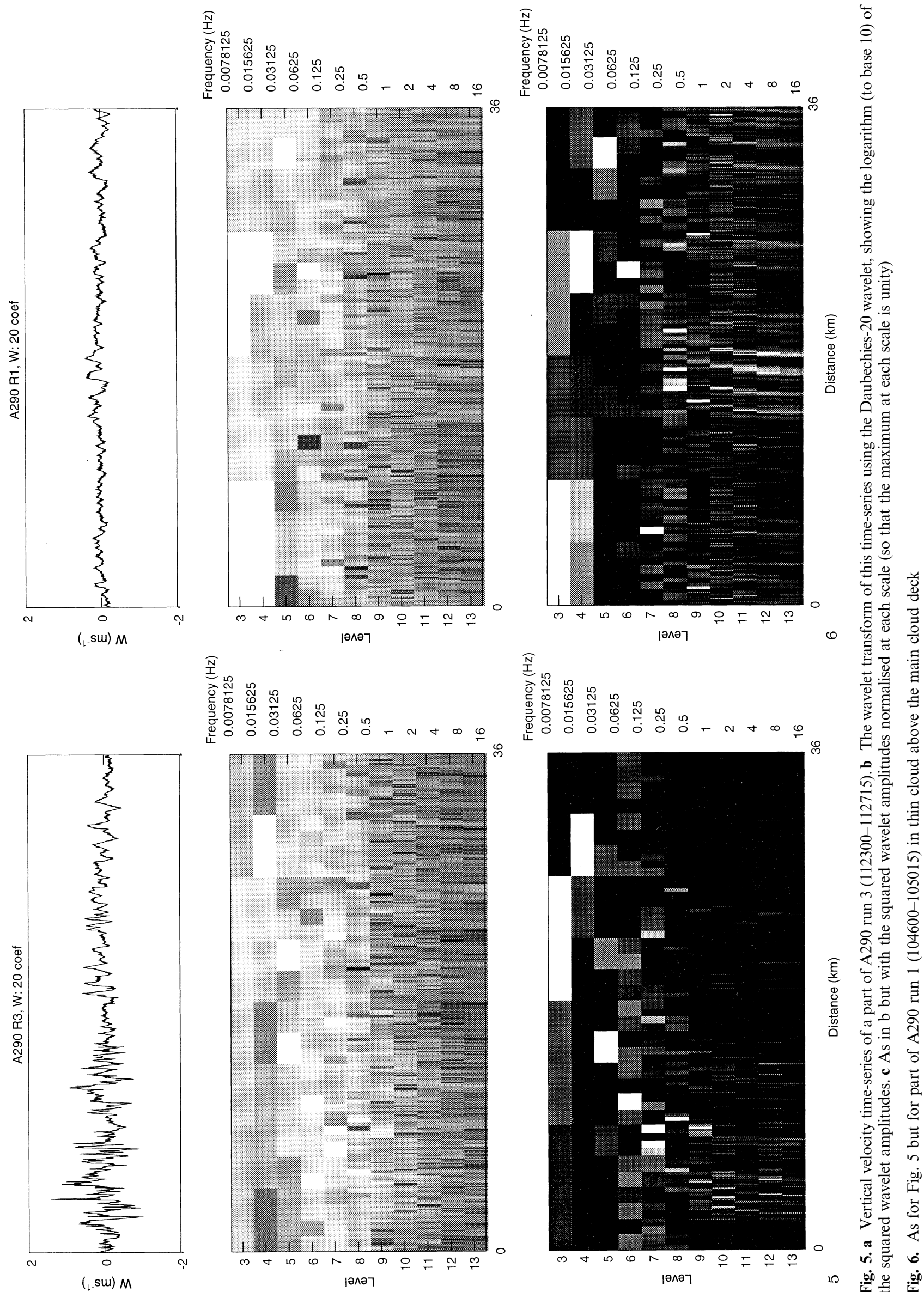

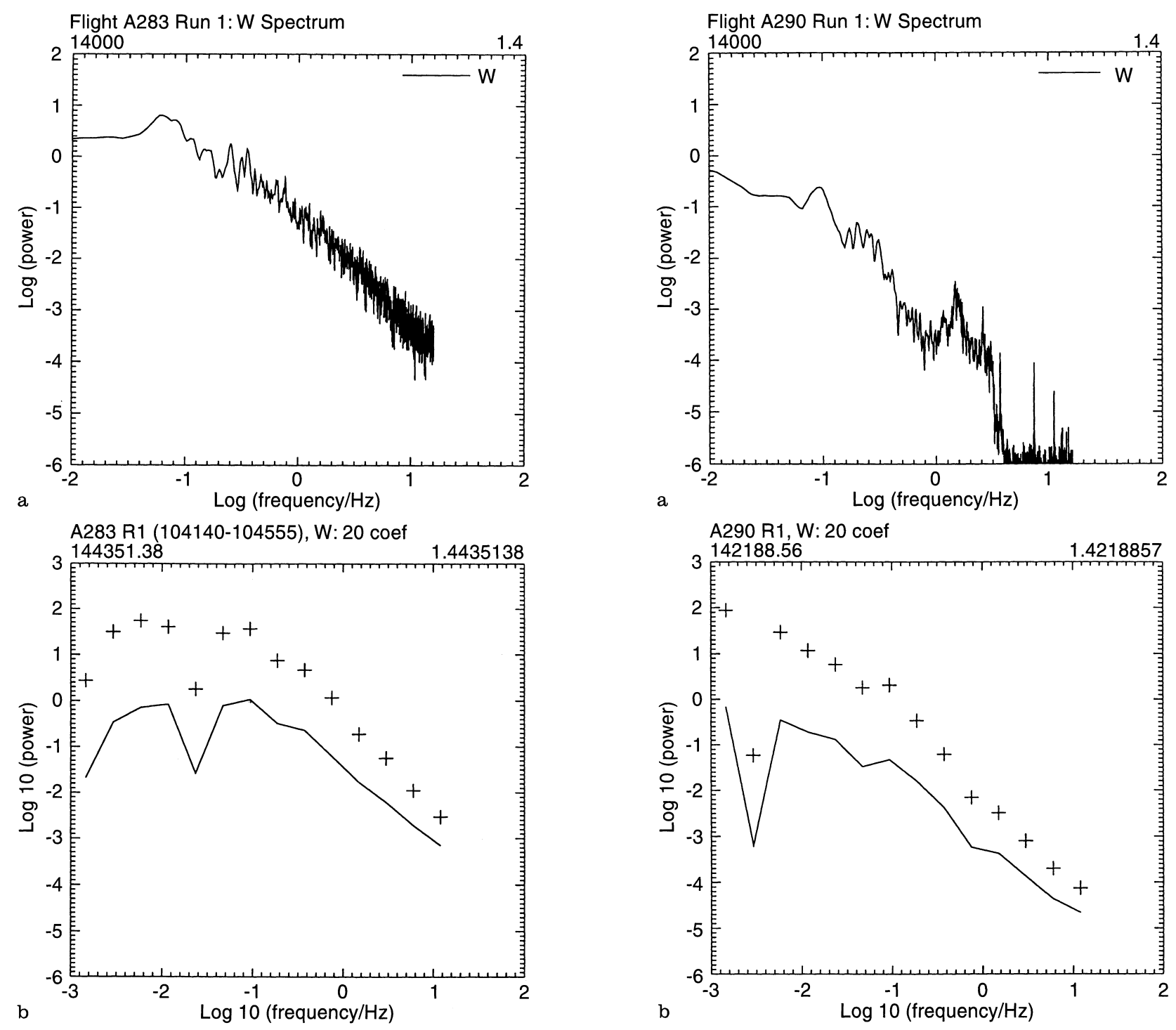

Fig. 7. a Fourier spectrum for the data shown in Fig. 3 (A283 run 1). The $x$-axis shows the base- 10 logarithm of the frequency $(\mathrm{Hz})$, and the $y$-axis shows the base- 10 logarithm of the spectral density. The numbers at the top of the diagram give the scales at the plot limits. b As for a, but for the average wavelet spectrum. The crosses show the average energy at each scale plus the standard deviation of the energy over the time-series. Note that the axes are slightly different, with the $x$-axis extending down to -3 instead of just -2 , and the $y$-axis up to 3 instead of 2

Fourier analysis is good for identifying the largerscale peaks due to convective activity or wave-like motions. However, it fails to show smaller-scale peaks due to local events unless the time-series used is short enough so that the local phenomenon takes up a reasonable fraction of the length of the time-series.

Wavelet analysis can also be used to identify processes which produce energy at larger scales, but have the added advantage that smaller-scale localised processes can also be identified along with their position in the time-series. It is useful for picking out localised regions where energy is produced at smaller scales, as it shows the energy contained in short pulses due to the finite nature of the basis functions. It could therefore be

Fig. 8. As in Fig. 7 but for the data shown in Fig. 6 (A290 run 1)

used in combination with conditional sampling techniques that pick out regions of enhanced shear or convection, for example, to produce an average energy spectrum for just those regions.

The limitation of this technique is the octave frequency representation. The effect of any single frequency is distributed over a range of frequencies. Translating the data set changes the representation of the wavelet transform. However, this gives the advantage that features in the data cannot be over-represented. This was shown for flight A290, where the 100-m peak seen in the Fourier spectrum was still seen in the wavelet spectrum but the magnitude was smaller. This was also noted by Gollmer et al. (1995), who used Daubechies-8 wavelets.

An alternative is to use a continuous wavelet transform using the Mexican Hat wavelet, for example. This has better frequency resolution but is computationally less efficient. The Daubechies wavelets seem to give sufficient results in these cases. 
Acknowledgements. The authors are indebted to the aircrew and scientists of the Meteorological Research Flight for their dedication in making the measurements and for their assistance in the post-flight analysis of the data. Support for this work from the CEC and the NERC is gratefully acknowledged.

Topical Editor L. Eymard thanks S. Bakan for his help in evaluating this paper.

\section{References}

Daubechies, I., Orthonormal bases of compactly supported wavelets, Commun. Pure Appl. Math., 41, 901-996, 1988.

Dmitriev, V. K., T. P. Kapitanova, V. D. Litvinova, N. G. Pinus, G. A. Potertikova, and G. N. Shur, Mean and microscale structure of wind and temperature fields in jet stream cirrus, in Proc. 9th Int. Cloud Phys. Conf. 2, Ed. Tallinn, Estonia pp. 347-350, 1984.

Gollmer, S. N., Harshvardan, R. F. Cahalan, and J. B. Snider, Windowed and wavelet analysis of marine stratocumulus cloud inhomogeneity. JAS, 52, 3013-3030, 1995.

Meneveau, C., Analysis of turbulence in the orthonormal wavelet representation, J. Fluid Mech., 232, 469-520, 1991.
Newland, D. E., An introduction to random vibrations, spectral and wavelet analysis (3rd Edn.), Longman Scientific and Technical, Harlen, England, 1993.

Nicholls, S., Measurements of turbulence by an instrumented aircraft in a convective atmospheric boundary layer over the sea, Q. J. R. Meteorol. Soc., 104, 653-676, 1978.

Press, W. H., S. A. Teukolsky, W. T. Vetterling, and B. P. Flannery, Numerical recipes in fortran. The art of scientific computing (2nd Edn), Cambridge University Press, Cambridge, 1992.

Quante, M., and P. R. A. Brown, Turbulence characteristics for different types of cirrus clouds, in Proc. 11th Int. Conf on Clouds and Precipitation, 1, Ed. Montreal, Canada pp. 510-513, 1992.

Quante, M., P. R. A. Brown, R. Baumann, B. Guillemet, and P. Hignett, Three-aircraft intercomparison of dynamical and thermodynamical measurements during the pre-EUCREX campaign, Contrib. Atmos. Phys. tropospheric profiling, 69, 129-303, 1995.

Slingo, A., S. Nicholls, and J. Schmetz, Aircraft observations of marine stratocumulus during JASIN, $Q$. J. R. Meteorol. Soc., 108, 833-856, 1982.

Smith, S. A., and P. R. Jonas, Observations of turbulence in cirrus clouds, Atmos. Res., 43, 1-32, 1997. 